\title{
Convection and the Extracellular Matrix Dictate Inter- and Intra- Biofilm Quorum Sensing Communication in Environmental Systems
}

\author{
Chuan Hao Tan, ${ }^{\dagger \dagger}$ Hyun-Suk Oh, ${ }^{\dagger \dagger}$ Vivek M. Sheraton, ${ }^{\dagger \dagger}$ Emiliano Mancini, Say Chye Joachim Loo,
} Staffan Kjelleberg, Peter M. A. Sloot,* and Scott A. Rice*

Cite This: Environ. Sci. Technol. 2020, 54, 6730-6740

Read Online

ACCESS

Wll Metrics \& More

Article Recommendations

Supporting Information

ABSTRACT: The mechanisms and impact of bacterial quorum sensing (QS) for the coordination of population-level behaviors are well studied under laboratory conditions. However, it is unclear how, in otherwise open environmental systems, QS signals accumulate to sufficient concentration to induce QS phenotypes, especially when quorum quenching (QQ) organisms are also present. We explore the impact of QQ activity on QS signaling in spatially organized biofilms in scenarios that mimic open systems of natural and engineered environments. Using a functionally differentiated biofilm system, we show that the extracellular matrix, local flow, and QQ interact to modulate communication. In still aqueous environments, convection facilitates signal dispersal while the matrix absorbs and relays signals to the cells. This process

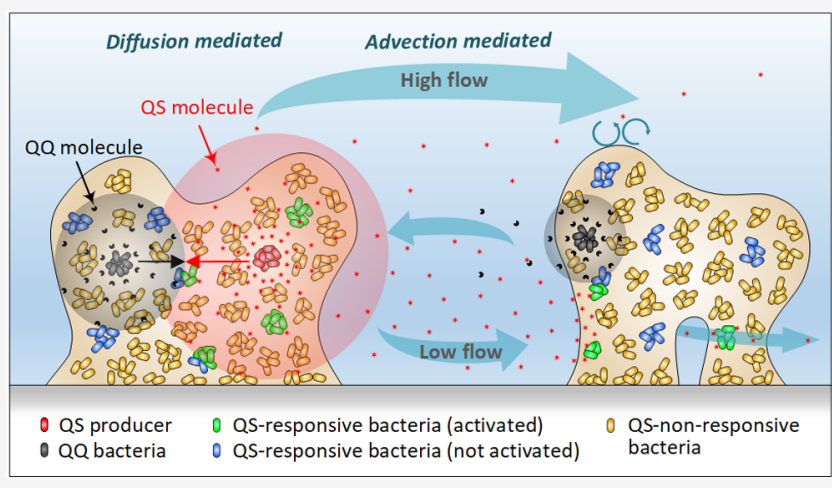
facilitates inter-biofilm communication even at low extracellular signal concentrations. Within the biofilm, the matrix further regulates the transport of the competing QS and QQ molecules, leading to heterogenous QS behavior. Importantly, only extracellular QQ enzymes can effectively control QS signaling, suggesting that the intracellular QQ enzymes may not have evolved to degrade environmental QS signals for competition.

\section{INTRODUCTION}

Quorum sensing (QS) is a microbial communication system that relies on the production, secretion, and perception of small diffusible signaling molecules. This system has evolved to control social behaviors that influence the fitness of microbial populations. ${ }^{1,2}$ The genetics and the regulatory pathways of many QS systems are well-documented, and the means by which such cooperative traits are protected from exploitation by QS cheaters have also been described. ${ }^{3-6}$ The prevailing view is that QS signaling allows populations of cells to assess their density according to the signals accumulated extracellularly. However, many environmental factors, other than cell density, such as mass transport, ${ }^{7,8}$ confinement, ${ }^{9-11}$ and signal degradation $^{12,13}$ can influence extracellular signal accumulation and hence a QS response. It has been shown that small numbers of intracellular Staphylococcus aureus can undergo QS, ${ }^{14,15}$ while modeling ${ }^{16}$ and in vitro single cells confinement ${ }^{9}$ studies further indicated that as few as $1-2$ cells/compartment can initiate high-density $Q S$ behaviors, including virulence and metabolic pathways needed for survival and growth in a host. This may contrast the requirement of large number of bacteria $\left(1 \times 10^{7}\right.$ to $\left.1 \times 10^{9}\right)$ typically observed for QS induction in macroscopic culture volume. ${ }^{17}$ Despite these established laboratory models in well-mixed cultures or in microfluidic systems, we have very limited information on how QS is achieved in spatially organized, species-rich microbial communities in natural and engineered environments. Although QS has been implicated in the formation of complex, mixed species granular biofilms and sludge biocakes in bioreactor ecosystems, ${ }^{18,19}$ QS signals in the liquid phase frequently occur at concentrations (i.e., $\sim 500 \mathrm{pM})^{19}$ that are significantly lower than the typical QS thresholds recorded for planktonic cultures (i.e., $10 \mathrm{~s}$ to $1000 \mathrm{~s} \mathrm{nM}) .^{7-9}$ Hence, it is unclear how biofilm communities may communicate via QS signals and achieve QS regulatory control at such low extracellular concentrations. Furthermore, the levels of QS signals can be influenced by quorum quenching (QQ) organisms that coexist with the QS signal producers in the community. For example, the presence of QQ activity has been shown to delay the formation of granular biofilms ${ }^{20}$ to quench QS-dependent bacterial infections ${ }^{21,22}$ and to control biofouling in membrane bioreactors. ${ }^{18}$ Given $\mathrm{QS}$ and QQ organisms commonly co-occur in nature, ${ }^{23,24}$ the interplay between the

Received: February 4, 2020

Revised: April 23, 2020

Accepted: May 10, 2020

Published: May 10, 2020 
QS and QQ activities is likely to have important impacts on the behavior of biofilm communities.

Within biofilms, microorganisms often grow as spatially structured cell clusters encased in an extracellular matrix of polymeric substances (EPS). ${ }^{19,25-27}$ The EPS matrix thus serves not only as a protective structure enabling biofilm cells to survive, but it can also partition different populations or communities within the environment. ${ }^{28}$ In monospecies cultures, biofilm thickness and the abundance of the EPS matrix have been associated with the ability of the biofilm to retain signals for QS induction under flow conditions, ${ }^{8}$ and the EPS components have been shown to influence communication between aggregates of biofilm populations. ${ }^{29-32}$ The impact of spatial distribution of aggregates of the biofilm on bacterial QS has also been addressed through modelling and microencapsulation technologies. ${ }^{7-9}$ However, little is known as to how the extracellular matrix impacts QS signaling for microbial consortia with different functions (i.e., QS vs QQ) and how spatial distribution and organization of individual competing populations, as segregated by the EPS matrix, may determine the outcome of communication within biofilm communities.

To address this knowledge gap, we have developed an experimental system to mimic QS processes reflective of open systems of naturally occurring biofilms that have functionally distinct community members. ${ }^{19,27}$ In this biofilm system, each bacterial population was encapsulated within a microliter-scale hydrogel granule and grown into a multicellular aggregate. By patterning these engineered aggregates onto an agar surface, we defined how the spatial organization of functionally distinct organisms impacts the interaggregate communication in different ecological contexts, including the presence and absence of local flow, using both experimental and numerical models.

\section{MATERIALS AND METHODS}

Engineering of Multicellular Aggregates Using Alginate Polymers. Sodium alginate solution was prepared by autoclaving $1.3 \% \mathrm{w} / \mathrm{v}$ sodium alginate (Thermo Fisher Scientific, Singapore). Overnight cultures, that is, QS signal producer (Escherichia coli EsaI), quencher (E. coli AiiO), and reporter (E. coli JB525), were washed three times with Milli-Q water. Thirty microliters of each bacterial culture was mixed with $970 \mu \mathrm{L}$ of sodium alginate solution $(1.3 \% \mathrm{w} / \mathrm{v})$ to reach a final $\mathrm{OD}_{600}$ of 0.25 . Approximately, $4 \mu \mathrm{L}$ of alginate-bacteria suspension was added dropwise, via a syringe needle (26G), into a sterile $\mathrm{CaCl}_{2}(4 \% \mathrm{w} / \mathrm{v})$ (Merck, Singapore) solution to crosslink the alginate polymers. The Ca-alginate-bacteria beads, with an average diameter of $1.92 \pm 0.04 \mathrm{~mm}$, were formed in $\mathrm{CaCl}_{2}$ solution after incubation for $20 \mathrm{~min}$ and washed twice with Milli-Q water (Figure S1A). To prevent any leakage of QS signal producers or quenchers, the alginate beads of QS signal producers or quenchers were coated with an additional layer of $\mathrm{Ca}$-alginate with a thickness ranging from 0.03 to $0.07 \mathrm{~mm}$ (Figure S1B). These Ca-alginate-bacteria beads were grown into multicellular aggregates in nutrient media as described below. The bacterial strains used in this study are described in Table S1.

Arraying Multicellular Aggregates into a Microbial Biofilm System. The Ca-alginate-bacteria beads were arrayed onto a $9 \mathrm{~cm}$ Petri dish, containing $1 \% \mathrm{w} / \mathrm{v}$ agarose hydrogel (with a thickness of $2.5 \mathrm{~mm}$ ) to construct a biofilm system, using the Ca-alginate gelation method. Briefly, $1 \mu \mathrm{L}$ of sodium alginate solution $(1.3 \% \mathrm{w} / \mathrm{v})$ was added dropwise evenly onto the agarose hydrogel according to the grid at an interval of 1 $\mathrm{cm}$. The Ca-alginate-bacteria beads were placed onto the sodium alginate drops, and $3 \mu \mathrm{L}$ of $\mathrm{CaCl}_{2}(4 \% \mathrm{w} / \mathrm{v})$ solution was added to each bead to allow crosslink formation between the bead and the agarose for $10 \mathrm{~min}$. Twenty milliliters of $10 \%$ w/v lysogeny broth (Lennox, Difco, Singapore), with or without $1 \% \mathrm{w} / \mathrm{v}$ agarose supplementation, was overlaid onto the plate to represent the hydrogel matrix or the aqueous environment, respectively. $\mathrm{CaCl}_{2}(0.095 \% \mathrm{w} / \mathrm{v})$ was supplemented to each medium to prevent swelling of the beads during the experiment.

Imaging and Image Processing. The plate containing the microbial biofilm system was placed inside a Gel-Doc XR+ system (Bio-Rad Laboratories, Singapore). UV fluorescent images were captured every $15 \mathrm{~min}$ with $20 \mathrm{~s}$ exposure for the aqueous environment experiment using a green fluorescent protein (GFP) emission $(520 / 530 \mathrm{~nm})$ filter. The exposure time was reduced to $5 \mathrm{~s}$ for the hydrogel matrix experiment to minimize the background fluorescence, which was observed to increase linearly with time. The raw data were exported as $1392 \times 1040$ pixel $(91.2 \times 91.2 \mu \mathrm{m}$ pixel size $) 16$-bit TIFF images for image analysis. To quantify the fluorescence intensity for each reporter aggregate, we first created a binary template image by localizing every reporter aggregate to a new layer using the "Pencil Tool" (size $20 \mathrm{px}$ ) of Photoshop software (Adobe, USA) (Figure S11). The template image was used to determine the mean grey value of each reporter aggregate using the "Analyse Particles" command of ImageJ (version 1.48, National Institute of Health, USA). After normalizing the mean grey value of each reporter aggregate by deducting the background fluorescence over time, the fluorescence intensity of each aggregate relative to a fully QS-activated aggregate (i.e., the relative fluorescence intensity) was determined. The overall shape of the synthetic aggregate and microcolonies of QS signal reporter strain developed within the synthetic aggregate was visualized using a Stereomicroscope (Stereo Discovery V8, Zeiss, Singapore) and a confocal laser scanning microscope (CLSM, LSM 780, Zeiss, Singapore), respectively.

Numerical Model. We modeled the transport of QS signals (i.e., AHL) and QQ enzymes (i.e., AiiO) using advection-diffusion-reaction equations. Details of numerical model and determination of QS activation thresholds are described in the Supporting Information. Briefly, the minimum activation concentration $C_{\mathrm{OS}}^{*}$ was obtained from the experimental data (Table. S2). Similarly, the time delay in granule activation $t_{\text {delay }}$ after reaching the minimum activation concentration was also obtained from the experimental data. The model parameters such as $D_{\mathrm{AHL}}, D_{\text {AiiO }}$, and $q_{\text {leak }}$ were tuned using three independent experiments to obtain their values. These model parameters were validated using different experimental scenarios.

QS Signal Absorption, Retention, and Release by the Extracellular Matrix. Alginate beads without bacterial cells were prepared as described above. The alginate beads were exposed to AHLs at final concentrations of $100 \mathrm{nM}$ for $1 \mathrm{~h}$ at room temperature. DMSO, which used to dissolve AHLs, was used as a solvent control. After brief washing in Milli-Q water for $5 \mathrm{~min}$, the alginate beads were added to a reporter assay, that is, JB525, to represent the matrix sample while the "washout" samples represent the supernatant. Relative fluorescent intensity was determined at 488/510 nm and 
normalized by OD600 for each sample using a microplate reader (Tecan Infinite M200pro, Switzerland). The experiment was repeated three times.

Statistical Analysis. All statistical analyses were performed using Prism (GraphPad version 6). Multiple $t$-tests with corrections using the Holm-Sidak method were performed to compare the QS signal retention by the alginate matrix to the supernatant. The significance levels for the family of comparisons were set at 5\%. The corrected $P$ values were reported. Data in all figures show means \pm SD $(n=3-6$, biological replicates).

\section{RESULTS}

Designing a Microbial Biofilm System for QS Study. An experimental biofilm system composed of individual populations of QS signal producers, responders, and quenchers was assembled to study the impact of the extracellular matrix, hydrodynamics, and signal interference on QS signaling at a macroscale level (i.e., centimeters) (Figure 1). To minimize confounding variables that potentially arise from interactions between different bacterial species, we engineered the QS and QQ functions into different populations of E. coli (Table S1). The signal producer was an E. coli strain harboring a p TrcHis2 plasmid with an acyl-homoserine lactone (AHL) signal synthase, esaI, derived from Pantoea stewartii, ${ }^{20}$ while the signal quencher strain encoded aiiO, an $\mathrm{AHL}$ lactonase from Ochrobactrum anthropi. ${ }^{33}$ Both esaI and aiiO genes were fused to a $P_{\text {trc }}$ promoter and constitutively expressed in all experimental conditions. EsaI synthesizes $N$-(3-oxohexanoyl)L-homoserine lactone (3OC6-HSL) while AiiO degrades a wide spectrum of AHLs, including 3OC6-HSL. ${ }^{33,34}$ E. coli JB525 is a signal reporter strain that is unable to produce AHLs but detects them with high sensitivity (e.g., 3OC6-HSL at 1 $\mathrm{nM}$ ) and expresses the GFP in a signal concentrationdependent manner. ${ }^{35}$ To simulate densely packed multicellular aggregates (i.e., biofilms), such as aerobic and anaerobic granules in wastewater treatment systems (Figure 1A), individual bacterial strains were encapsulated using alginate polymers to produce aggregates of cells (i.e., granules) with an average dimension of $1.91 \pm 0.06 \mathrm{~mm}$ (Figures $1 \mathrm{~B}$ and S1A,B). Bacterial microcolonies, similar to microbial clusters found in naturally occurring biofilms, ${ }^{36,37}$ were formed within the alginate matrix after $24 \mathrm{~h}$ incubation at room temperature (Figure 1C). Using these strain-defined aggregates (defined as producers, responders, and quenchers), we built a microbial biofilm system on the surface of an agarose hydrogel $(1 \% \mathrm{w} / \mathrm{v})$ where the spatial coordinates of each aggregate population were defined along a grid (Figure 1D). Unless otherwise stated, bacterial aggregates were placed $1 \mathrm{~cm}$ apart from one another (in coordination $x$ and $y$ ). Nutrients for growth were supplied in an agarose hydrogel $(1 \% \mathrm{w} / \mathrm{v})$, representing the extracellular matrix, or in a liquid medium, characteristic of the aqueous environments (Figure $1 \mathrm{~F}$ ). The biofilm was cultured in a dark-chamber, and signaling (activation of the QS reporters, i.e., QS activation) was visualized under UV illumination using a camera equipped with a GFP emission filter (Figure 1E). This setup represents a platform for studying species interactions based on diffusible molecules, such as AHLs and QQ enzymes, in natural communities.

QS Signaling in a Hydrogel Matrix. To simulate QS signaling in natural biofilms such as microbial mats, where the organisms are embedded in an extracellular matrix, we used $1 \%$ agarose hydrogel $(\mathrm{w} / \mathrm{v})$ as the biofilm matrix of the microbial

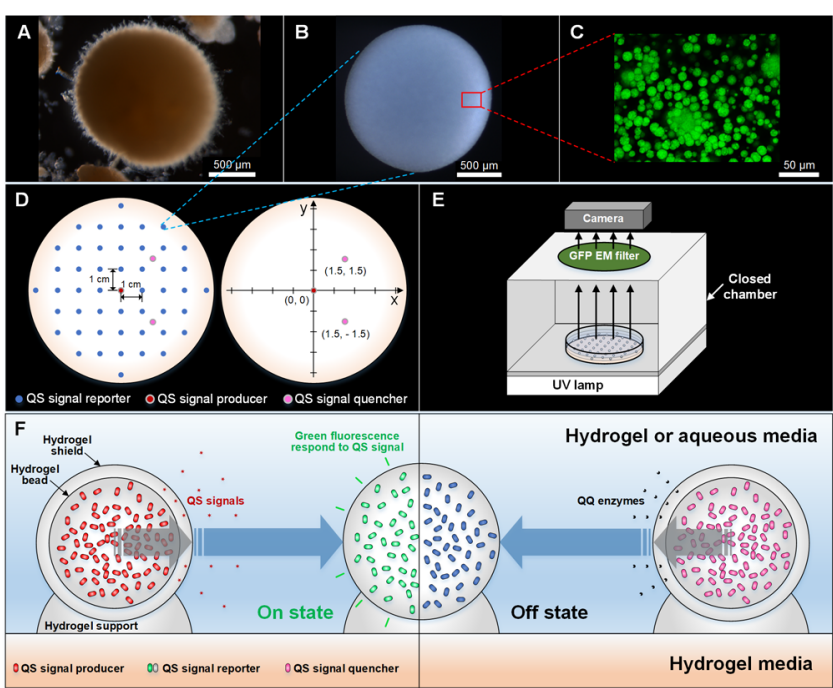

Figure 1. Experimental configuration. (A) Natural multicellular aggregate-a highly structured, multispecies microbial community in the wastewater treatment plant/bioreactor. (B) Synthetic multicellular aggregate-a biofilm model engineered by encapsulating individual strains into an alginate-based hydrogel bead. (C) Microcolonies of QS signal reporter strain developed within an alginate-based hydrogel bead after incubating for $24 \mathrm{~h}$ at room temperature. The reporter aggregate was exposed to $100 \mathrm{nM}$ of QS signal (i.e., 3OC6-HSL) for $4 \mathrm{~h}$ for GFP induction prior to imaging by CLSM at $488 / 510 \mathrm{~nm}$. (D) Layout of a microbial biofilm system composed of individual aggregates of QS signal reporters (blue), producers (red), and quenchers (pink) on a $9 \mathrm{~cm}$ Petri dish. (E) The setup for time series snapshots of signaling events in the experimental system. The GFP fluorescence, representing QS activation, is visualized under UV illumination using a camera equipped with a GFP emission filter in an enclosed chamber. (F) Illustration of the cross-sectional view of a microbial biofilm system setup on a $9 \mathrm{~cm}$ Petri dish. The aggregates were crosslinked onto an agarose hydrogel layer $(1 \% \mathrm{w} / \mathrm{v})$ and overlaid with $10 \%$ lysogeny broth $(\mathrm{w} / \mathrm{v})$, with or without $1 \%$ agarose $(\mathrm{w} / \mathrm{v})$ supplementation, to represent the hydrogel matrix or the aqueous environment, respectively. Both QS signal producer (red) and quencher (pink) aggregates were protected with an additional layer of alginate (i.e., hydrogel shield) to prevent cell leakage. QS signals and QQ enzymes are expected to be released from the respective source of aggregates into extracellular space to activate the QS signal reporters (green-on state) or to inhibit reporter activation (blue-off state) by degrading the QS signals. The image for the natural multicellular aggregate $(\mathrm{A})$ is adapted from Tan et al. ${ }^{19}$

system (Figure 2). In this experiment, AHLs were secreted from two spatially separated, actively growing signal-producing aggregates located at positions $(1,1)$ and $(0,-2)$ (Figure $2 \mathrm{~A})$. Activation of the surrounding reporter populations was uniform and symmetric. Individual reporter aggregates were sequentially activated, where the signal acquisition time for each reporter increased with their distance from the AHL producer, which is a reflection of signal concentration and diffusion rates. To elucidate the mechanism responsible for this pattern of induction, we developed a three-dimensional (3D) in silico model that integrated simple AHL diffusion with the dynamic signal production rate. This incorporated the logistic growth of the AHL producer, the abiotic signal degradation rate, as well as cooperative activation behavior of the reporter (Figures 2B, S1C,D and see Numerical Model in the Supporting Information). The model predicted the spatiotemporal changes in signal profiles, showing localized AHL accumulation around the producer (Figure 2C) as well as 


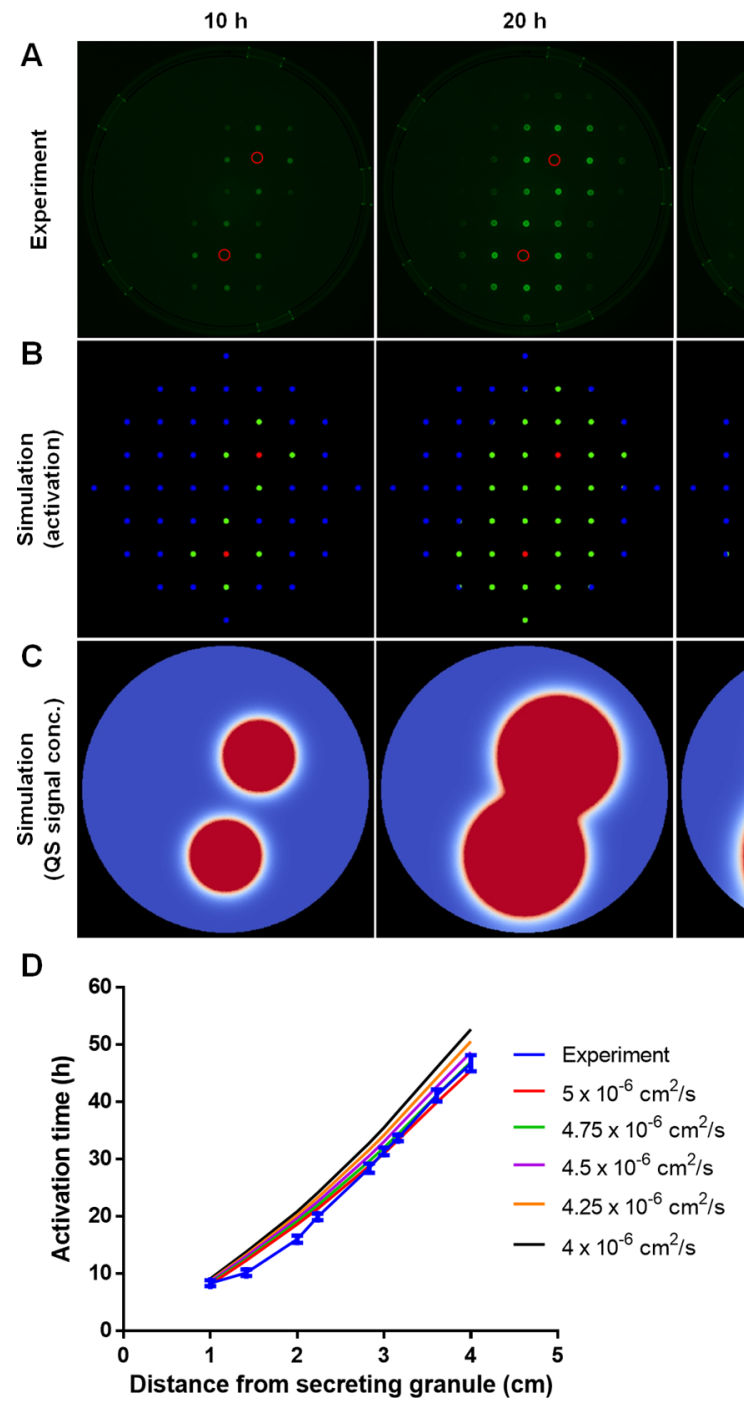

$30 \mathrm{~h}$
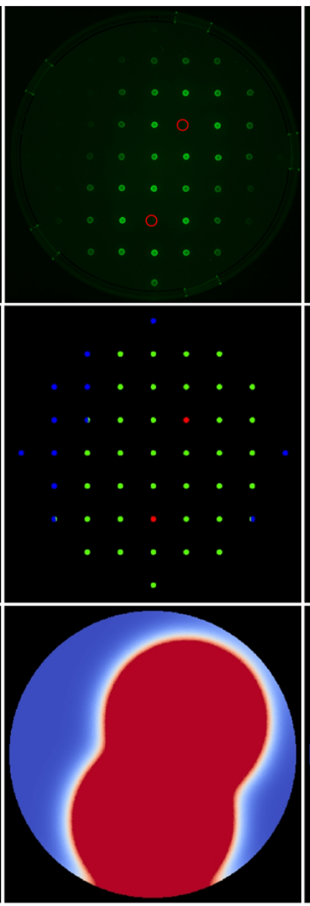

E
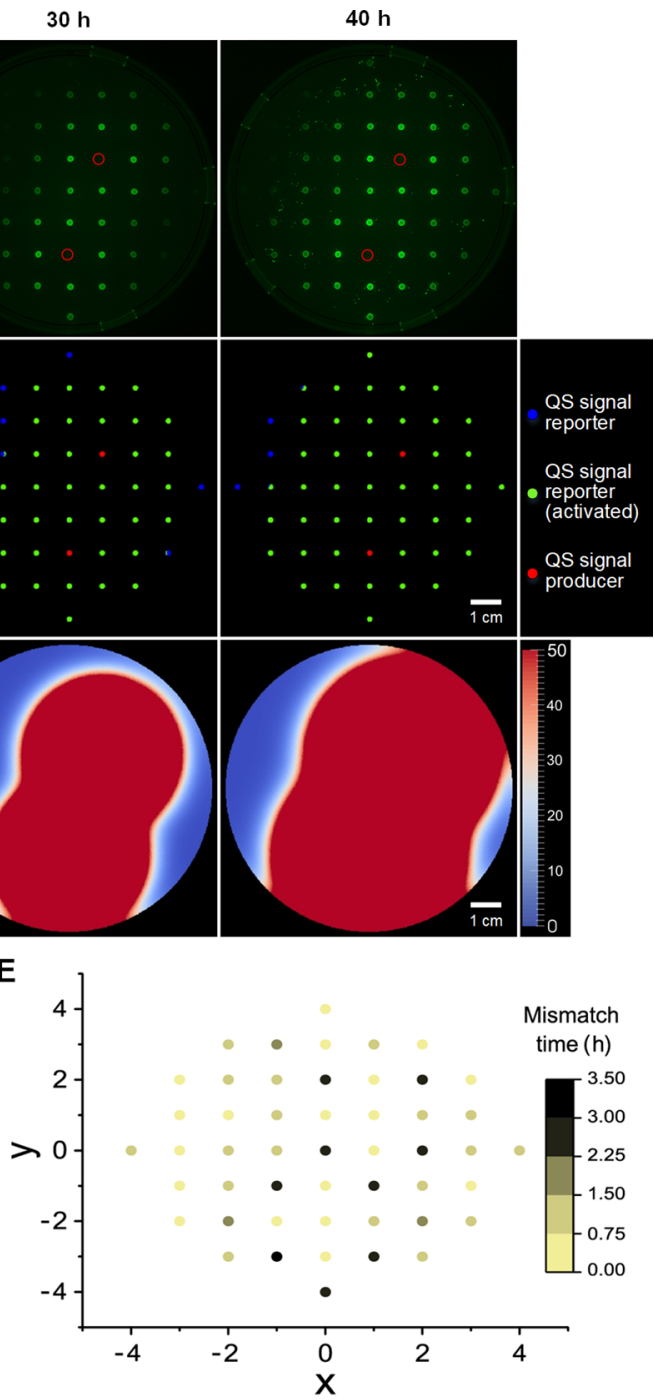

Figure 2. QS signaling in a hydrogel matrix. (A) Activation of QS signal reporter aggregates, that is, GFP induction, in response to signals generated by QS signal producer aggregates (open red circles) located at $(1,1)$ and $(0,-2)$. (B) Simulations of QS activation based on the signal diffusivity $\left(D_{\mathrm{AHL}}\right)$ of $4.75 \times 10^{-6} \mathrm{~cm}^{2} / \mathrm{s}$. (C) Spatio-temporal prediction for the QS signal concentration profile based on the signal diffusivity of $4.75 \times 10^{-6} \mathrm{~cm}^{2} / \mathrm{s}$. (D) Simulations of spatio-temporal QS responses based on different QS signal diffusivities. Means \pm SD are presented $(n=3)$ for the experiment. (E) The mismatch of QS activation times for the experimental results and the simulations with a signal diffusivity of $4.75 \times 10^{-6}$ $\mathrm{cm}^{2} / \mathrm{s}$.

limited signal diffusion away from the producer within biofilms. Diffusivity of the AHL was the only free parameter in this model and was tuned using experimental results that was performed with single QS signal producer bead located at $(0,0)$. Based on the simulations, the diffusivity of AHL $\left(D_{\mathrm{AHL}}\right)$ in the hydrogel matrix was determined to be $4.75 \times 10^{-6} \mathrm{~cm}^{2} / \mathrm{s}$ (Figure 2D), which is close to the estimated diffusivity of AHL in water, $4.9 \times 10^{-6} \mathrm{~cm}^{2} / \mathrm{s}$, reported previously. ${ }^{38}$ The difference in activation times between experiments and simulations at the same corresponding positions was calculated as absolute Fréchet distances. This measure denotes the expected time interval within which a simulated activation is bound to be observed in the experimental setting at a given location. The average relative error of mismatch in the QS activation time between the experiment (having two QS signal producer beads located at $(1,1)$ and $(0,-2)$ as shown in Figure $2 \mathrm{~A}$ ) and numerical simulations was approximately $6 \%$, and the Fréchet distance showed a maximum mismatch of 3.5 $\mathrm{h}$ (Figure 2E), indicating a good match between the experiment and the simulations.

QS Signaling in a Hydrogel Matrix in the Presence of Quorum Quenchers. To determine the impact of physical distribution of quorum quenchers on QS signaling in the biofilm context, two signal-quenching aggregates were placed in proximity to $(-0.5,-0.5$, representing the $x$ and $y$ coordinates, respectively, of the aggregates in the model system) or at a distance away from $(1.5,1.5)$ the signal producer $(0,0)$ (Figure 3$)$. Both quenchers exhibited localized QQ effects, and the activation of reporter aggregates was determined by the local concentration of QS signals affected by the distance from both the signal producer and the quencher.

AiiO has been reported to be a cytoplasmic QQ enzyme in the original host $O$. anthropi, ${ }^{39}$ and it was therefore assumed here that the $\mathrm{AiiO}$ remained as an intracellular protein when it is expressed heterogeneously in E. coli. Additionally, the production rate of $\mathrm{AiiO}$ was expected to be similar to that of EsaI because both enzymes were expressed using the same 


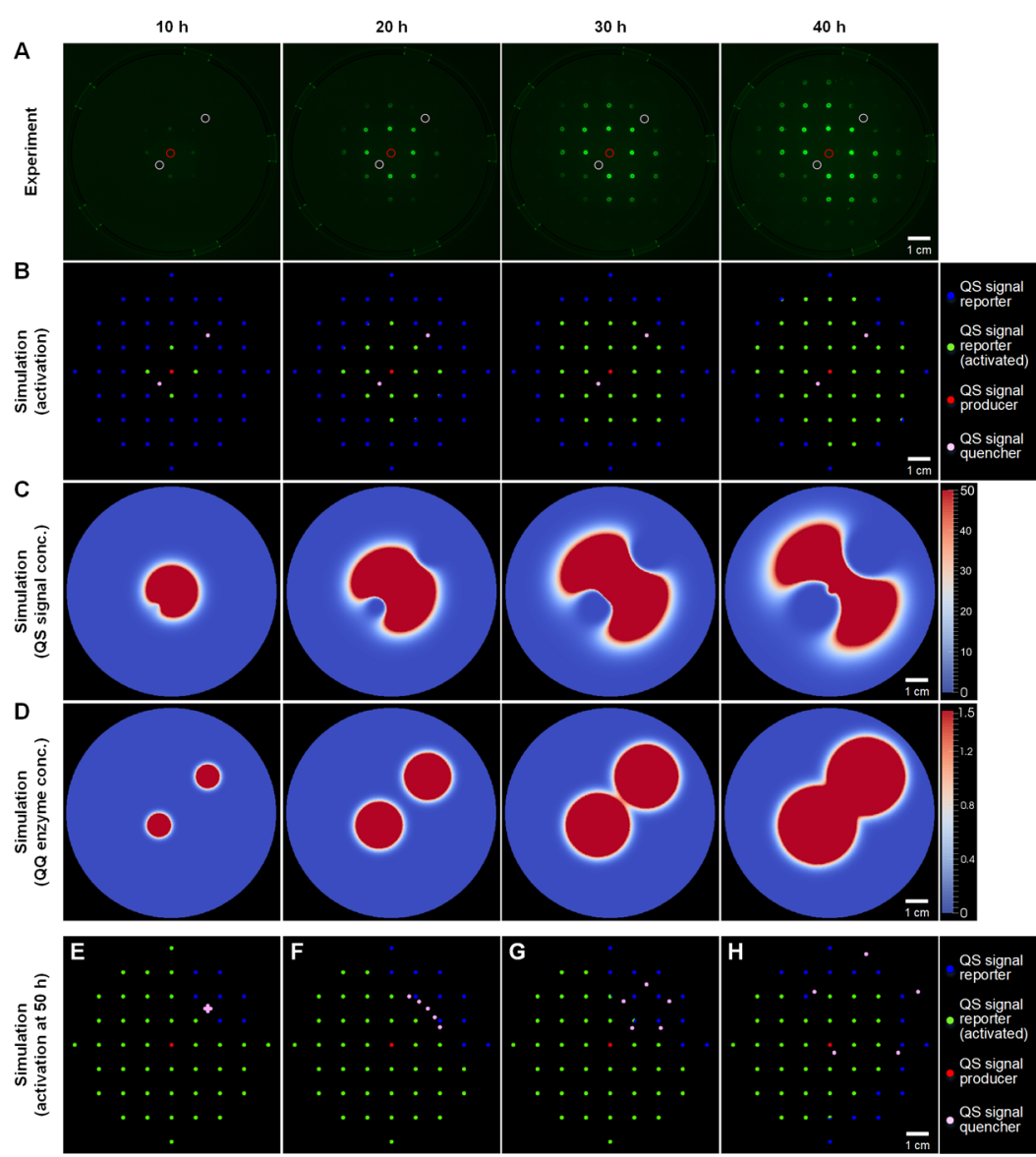

Figure 3. QS signaling in a hydrogel matrix in the presence of quorum quenchers. (A) Activation of QS signal reporter aggregates, that is, GFP induction, in response to signals generated by QS signal producer aggregate (open red circles) located at $(0,0)$ with the presence of QS signal quencher aggregates (open pink circles) located at $(1.5,1.5)$ and $(-0.5,-0.5)$. (B) Simulations of QS activation. (C) Spatio-temporal prediction for the QS signal concentration profile. (D) Spatio-temporal prediction for the QQ enzyme concentration profile. The diffusivities of QS signals $\left(D_{\mathrm{AHL}}\right)$, that is, $4.75 \times 10^{-6} \mathrm{~cm}^{2} / \mathrm{s}$ and QQ enzymes $\left(D_{\text {AiiO }}\right)$, that is, $1.35 \times 10^{-6} \mathrm{~cm}^{2} / \mathrm{s}$ were used in all simulations. (E-H) Simulation of QS signaling in a hydrogel matrix in the presence of quorum quenchers with different spatial configurations. Specifically, the QS signal quencher aggregates positioned with a geometric center at $(1.5,1.5)$ and vertices spaced equally from this center as $(\mathrm{E})$ concentrated, $(\mathrm{F})$ arc, $(\mathrm{G})$ small pentagon, and $(\mathrm{H})$ large pentagon configurations.

promoter and plasmid system. We therefore performed simulations assuming that no $\mathrm{AiiO}$ was released outside the quencher aggregates and that quenching of AHL occurred based on diffusion of the AHL into the quencher aggregates where they are degraded by AiiO in the cells. These simulations suggested that all reporter populations were activated after a certain period of time despite the presence of quenchers. Thus, the effect of QQ was predicted to be negligible, even when adjacent to the quencher aggregates (Figure S2). Because the experimental data showed QS inhibition, these results suggested that QQ activity was not limited to the intracellular/intra-aggregate process and that the enzymes may be released into the extracellular/extra-aggregate space. We subsequently confirmed the presence of extracellular QQ activity outside the quencher aggregate after incubating the aggregate in the growth medium for $6 \mathrm{~h}$ or more (Figure $\mathrm{S} 3 \mathrm{~A}, \mathrm{~B}$ ). The release of AiiO corresponded to the detection of the intracellular (periplasmic) enzyme alkaline phosphatase extracellularly, suggesting that AiiO might be released via cell lysis by a small subpopulation of cells or exported through outer membrane vehicles (Figure S3C). The incorporation of these findings into our existing model enabled an improved and more accurate simulation of the experimental observations of reporter activation with an average relative error of mismatch of approximately 6.5\% (Figures 3B, S4A and see Numerical Model in the Supporting Information). The model was subsequently tuned for two free parameters, diffusivity of AiiO and amount of AiiO released by cell lysis $\left(K_{\text {leak }}\right)$ using experimental results that was performed with two QS signal quencher beads located at $(1.5,1.5)$ and $(1.5,-1.5)$. The diffusivity of $\mathrm{AiiO}\left(D_{\mathrm{AiiO}}\right)$ in the hydrogel matrix was determined to be $1.35 \times 10^{-6} \mathrm{~cm}^{2} / \mathrm{s}$, which is approximately 3.6 times slower than the diffusivity of the AHL (Figure S5). The average relative error of mismatch in the QS activation time between the validation experiment (having two QS signal quencher beads located at $(1.5,1.5)$ and $(-0.5,-0.5)$ as shown in Figure $3 \mathrm{~A}$ ) and numerical simulations was approximately $6.6 \%$, and the Fréchet distance showed a maximum mismatch of $4.3 \mathrm{~h}$ (Figure S4), indicating a good match between the experiment and the simulations. Correspondingly, the model predicted minimal AiiO transport in the hydrogel matrix and hence allowed the build-up of the released enzyme around the quencher aggregate over time (Figure 3D). As a consequence, AHL concentrations around the quencher aggregate were predicted by the simulations to be significantly reduced compared to other regions (Figure 3C), although it 


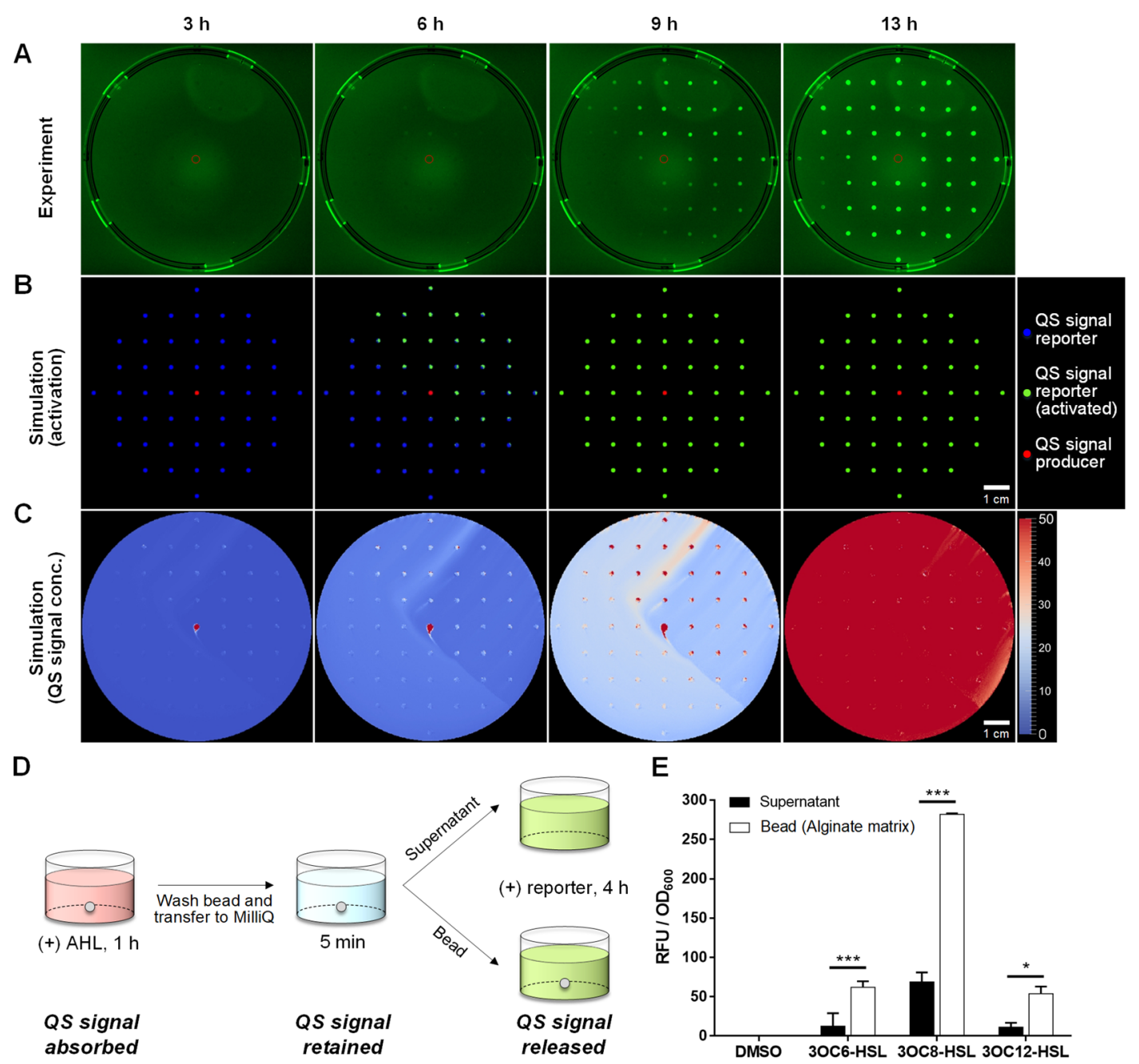

Figure 4. QS signaling in an aqueous environment. (A) Activation of QS signal reporter aggregates, that is, GFP induction, in response to signals generated by QS signal producer aggregate (open red circle) located at $(0,0)$. (B) Simulations of QS activation based on the Navier-Stokes model for fluid convection. (C) Spatio-temporal prediction for the QS signal concentration profile. (D) Experimental design to test for QS signal absorption, retention, and release by extracellular matrix (e.g., alginate bead). (E) GFP induction in the QS reporter strain by QS signals, including 3OC6-HSL, 3OC8-HSL, and 3OC12-HSL, released from supernatant or alginate bead. DMSO was used as a solvent control. Relative fluorescent intensity was determined at $488 / 510 \mathrm{~nm}$ and normalized by $\mathrm{OD}_{600}$ for each sample. Means $\pm \mathrm{SD}$ are presented $(n=3)$. Multiple $t$-tests with corrections using the Holm-Sidak method were performed to compare the QS signal retention by the alginate matrix to the supernatant, where significant differences are indicated as follows: $* P<0.01, * * * P<0.001$.

might still be sufficient to allow QS activation, depending on the relative distance to the signal producer (Figure 3A,B). Our numerical model further predicted that dispersed $Q Q$ populations would have a greater impact on QS behavior compared to the highly clustered QQ populations (Figure 3E$\mathrm{H})$. For example, $14 \%$ of the responder populations were inactive with the clustered QQ populations (Figure 3E) while up to $35 \%$ of the responders, depending on specific configurations, were inactive when the QQ populations were dispersed throughout the system (Figure 3F-H).

QS Signaling in an Aqueous Environment. In contrast to the highly organized, symmetrical QS activation pattern observed in the hydrogel matrix (Figure 2A), activation of QS in the open aqueous environment with boundaries (i.e., the aqueous media is facing the open air and confined within a Petri dish) was random and uneven (Figure 4A). The reporter populations were activated independent of their distance from the signal producer. The activation process often occurred unidirectionally, initiating from one edge of the plate with activation proceeding away from that edge until all of the reporter populations were activated. The entire reporter population could be fully activated by a single signal producer in $15.5 \mathrm{~h}$ (i.e., with a $6 \mathrm{~h}$ interval from the time the first aggregate was activated until all of the reporter aggregates were fully induced), while this might take more than $50 \mathrm{~h}$ in the hydrogel matrix model and setup (Figure S6A). Given the discrepancy in the QS activation times, the hydrogel-based numerical model was not able to reproduce the rapid QS induction profile in the aqueous environment, even if the signal production rate was increased 100 fold (Figure S7). For example, a 100 fold increase in the signal production rate only reduced the time to full QS activation by $18 \mathrm{~h}$ (i.e., from 52 to $34 \mathrm{~h}$ ). Thus, it was clear that the irregular and rapid QS activation in the aqueous environment could not be driven solely by diffusion but involves other modes of signal transport, including convection induced by evaporation of aqueous media or an air flow inside the chamber. The presence of a local flow induced by an ambient air flow in the enclosed incubation 


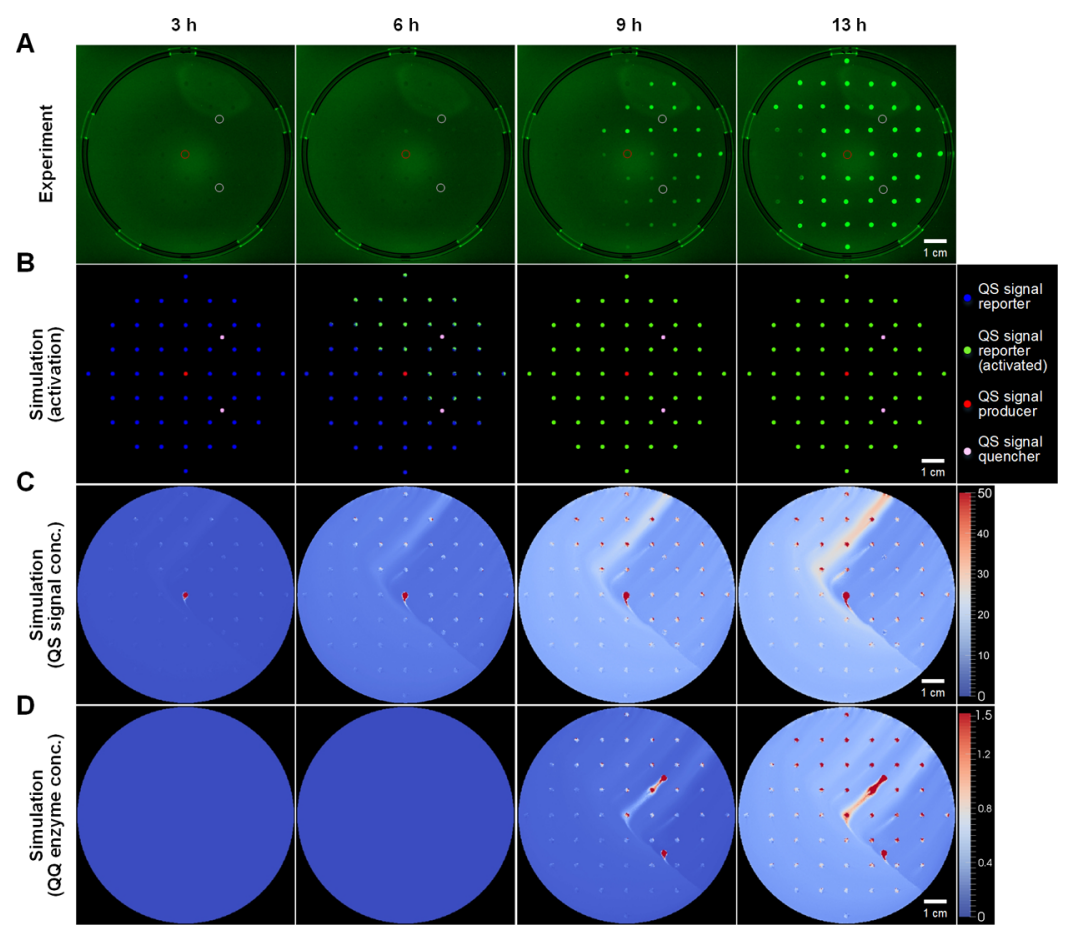

Figure 5. QS signaling in an aqueous environment in the presence of quorum quenchers. (A) Activation of QS signal reporter aggregates, that is, GFP induction, in response to signals generated by QS signal producer aggregate (open red circle) located at $(0,0)$ with the presence of QS signal quenchers aggregates (open pink circle) located at $(1.5,1.5)$ and $(1.5,-1.5)$. (B) Simulations of QS activation based on the on the Navier-Stokes model for fluid convection. (C) Spatio-temporal prediction for the QS signal concentration profile. (D) Spatio-temporal prediction for the QQ enzyme concentration profile.

chamber was verified by a drift assay (Figure S8A). When the Petri dish was covered with a lid, the local flow was prevented but evaporation-induced convection remained (Figure S8B). Correspondingly, the rate of QS activation was reduced remarkedly in the closed system compared to the open lid experiments (Figure S8C). For example, $88 \%$ of the responder populations were activated within $10 \mathrm{~h}$ in the open aqueous system (i.e., without lid) compared to only $37 \%$ in the closed aqueous system (i.e., with lid) and $8 \%$ in the open hydrogel system (i.e., without lid). By incorporating a convection term as a means of local flow and evaporation induction to the hydrogel based model, we simulated the experimental observations in the aqueous environment with velocity being the only free, tuneable parameter and found that an $x$-velocity of $0.1 \mathrm{~cm} / \mathrm{s}$ and $y$-velocity of $0.2 \mathrm{~cm} / \mathrm{s}$ qualitatively best reproduced the QS pattern (Figures 4B, S9 and see Numerical Model in the Supporting Information). Although it was not possible to precisely simulate the spatial QS activation profile in the experimental data, the simulations predicted similar activation times of $13.5 \mathrm{~h}$ for the entire system, with a $5 \mathrm{~h}$ interval from the time of activation for the first aggregate until all were induced, indicating a close match between experiments and the simulations.

The simulations subsequently predicted non-circular or asymmetrical signal concentration contours in the aqueous environment (Figure 4C). The signal accumulation was estimated to occur rapidly, and the entire system reached a minimum concentration of $50 \mathrm{nM}$ in $13 \mathrm{~h}$. Interestingly, many signal hot spots, where significantly higher signal concentrations accumulated compared to the immediate surroundings, were found across the system for the early time points (i.e., $<9 \mathrm{~h}$ ). The majority of these hot spots overlapped with the positions of the reporter aggregates, which were encapsulated in the alginate matrix, indicating that the signals may preferentially accumulate in, or are retained by, the matrix. Based on these outcomes, we further hypothesized that AHLs were absorbed by the biofilm matrix (e.g., alginate) from the aqueous environment leading to a higher AHL concentration in the bead over time to enable reporter activation within the matrix. To verify this hypothesis, we exposed alginate beads without bacteria to different AHL solutions, including 3OC6HSL, 3OC8-HSL, and 3OC12-HSL for $1 \mathrm{~h}$ (Figure 4D). After a brief washing, the alginate beads were added to a reporter assay to represent the matrix sample while the "washout" samples represent the supernatant. In all cases, the reporter strain was activated by signals released from the alginate matrix and the level of activation was significantly higher than that of the supernatant (i.e., $>5$ fold, $P<0.05$ for all) (Figure 4E), demonstrating the absorption/desorption of signals into the biofilm matrix. This outcome underscores a crucial role of the biofilm matrix in QS signaling in aqueous environments.

QS Signaling in an Aqueous Environment in the Presence of Quorum Quenchers. Unlike the case for the hydrogel matrix (Figure 3A), the addition of two signalquenching aggregates at positions $(1.5,1.5)$ and $(1.5,-1.5)$ in the aqueous environment did not alter the QS activation profile, with the exception of a limited number of reporter aggregates that remained partially inactive after $13 \mathrm{~h}$ incubation (Figure 5A). Such experimental observations were also predicted by the simulations (Figure $5 \mathrm{~B}$ and see Numerical Model in the Supporting Information). While the presence of QQ aggregates had little impact on the pattern of QS activation experimentally (Figures 5A vs 4A), the simulations suggested that the quenchers could strongly suppress the accumulation of high concentrations of the signal (i.e., $>10 \mathrm{nM}$ ) (Figures 5C vs 4C). For example, in the absence 

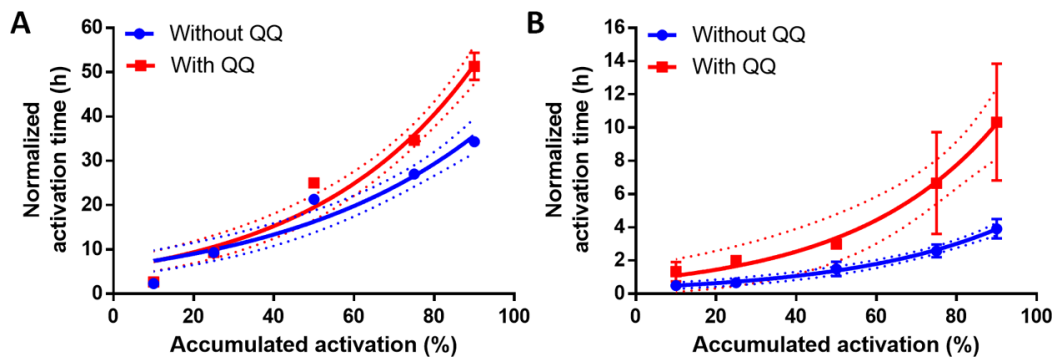

Figure 6. Extracellular matrix, hydrodynamics, and QQ regulate the pattern of QS signaling. Cumulative QS responses of the signal reporters embedded within (A) a hydrogel matrix and (B) an aqueous environment, determined in the absence (blue circle) or the presence of signal quenchers (i.e., QQ-red square) according to the experimental system layout shown in Figure 1d. The QS activation time was normalized by subtracting the time taken for the first reporter aggregate to be activated and the subsequent time spent for cumulative activation of the reporters in the system. Means \pm SD are presented $(n=3)$. The dotted lines represent the $95 \%$ confidence intervals.

of quenchers, the entire system was saturated with signals with a minimum concentration of $50 \mathrm{nM}$ by $13 \mathrm{~h}$ (Figure 4C), while in the presence of quenchers, the average signal concentration was predicted to be less than $15 \mathrm{nM}$ by $13 \mathrm{~h}$ (Figure 5C).

Extracellular Matrix, Hydrodynamics, and QQ Regulate the Pattern of QS Signaling. To quantitatively measure the impacts of the extracellular matrix, hydrodynamics, and QQ on QS signaling, we compared the cumulative $\mathrm{QS}$ responses of reporter populations in different environments. In these experiments, the spatial coordinates of individual populations in each system were standardized (Figure 1D). The activation time for each experimental condition was normalized by subtracting the time taken for the first reporter aggregate to be activated (Figure 6). In the hydrogel matrix, the normalized time for activating the first $50 \%$ of the reporter populations was almost identical (mean difference of $4 \mathrm{~h}$ ) for experiments with and without the quenchers (Figure 6A). However, the time for activation of $90 \%$ of the reporters was significantly longer in the presence of quenchers than in their absence (mean difference of $18 \mathrm{~h}$ ). In the aqueous environment, the difference in mean activation times between experiments with and without quenchers increased from less than $30 \mathrm{~min}$ to more than $6 \mathrm{~h}$ as the percentage of activated reporters increased from 15 to $90 \%$ (Figure 6B). These findings were consistent with the prediction of AHL profiles in both scenarios (Figures $4 \mathrm{C}$ and 5C), indicating a reduced, but global QQ impact on QS. Importantly, in the absence of quenchers, full QS activation in the aqueous environment could be achieved at least 10 times faster than that in the hydrogel matrix (Figure 6). Even in the presence of quenchers, the duration required to arrive at more than $90 \%$ of signal responder populations activated was approximately five times shorter than that in the hydrogel matrix, highlighting the extent by which the interaction with the physical environment may influence QS behavior.

\section{DISCUSSION}

The spatiotemporal activity of QS signaling and QQ is strongly influenced by the mode of mass transport and/or the biogeography and organization of individual populations in the biofilm system. First, monospecies biofilm studies have indicated the ability of biofilms to retain AHLs at concentrations 1000 fold higher than in the liquid phase, ${ }^{40}$ and the biofilm thickness can affect the levels of QS induction under flow conditions. ${ }^{8}$ Here, we further show that the EPS matrix of the biofilm controls molecular transport (e.g., QS signals and QQ enzymes) via diffusion mechanisms, resulting in distinct zones of QS signaling and QQ inhibition (Figures 2 and 3). These findings clearly support the hypothesis that QS would be most pronounced in the vicinity of QS organism/ population clusters within biofilms. ${ }^{41,42}$ Because the production of an EPS matrix by many organisms is directly regulated by QS, ${ }^{43-45}$ one might speculate that AHL-EPS synthesis/ secretion has co-evolved to facilitate cooperative QS behavior to immediate neighbors for population benefits and evolutionary stability of QS, according to the kin selection hypothesis. ${ }^{46,47}$ Similarly, QQ activity would be concentrated within biofilms and thus compete with QS signaling. It is important to note, however, that although the QQ function is localized within the biofilm matrix, the impact of QQ on the QS behavior may vary according to the spatial organization and distribution of different species. Specifically, we found that dispersed QQ populations have greater global influence on the QS behavior compared to the QQ populations present in the clusters (Figure $3 \mathrm{E}-\mathrm{H}$ ). Spatial organization is also an important factor in the control of QS activation. For example, QQ may not be sufficient to inactivate QS when the quenching organism is close to the source of signal production. However, the QQ may degrade a sufficient amount of the signal to prevent signal induction for responders that are also further away from the signal source (Figure 3A,B,H). These observations are likely due to the differences in production rate and diffusivity of the molecules. Hence, these findings suggest a mechanism for heterogenous QS activation in biofilm communities with functionally distinct populations, that is, QS and QQ based on the spatial organization of those functional populations.

In contrast, in an aqueous environment, the transport of molecules was mainly driven by convection, including the local flow and evaporation-induced advection. ${ }^{48}$ Although convection in open environment with no boundary, for example, in the flow cell system, dilutes signals and deters QS induction, ${ }^{8}$ we demonstrated here that convection in an open environment with boundaries mediates rapid AHL and AiiO dissemination and allows for their activity. For example, in the absence of quenchers, full $\mathrm{QS}$ activation in the aqueous environment, under the conditions used here, could be accomplished at least 10 times faster than that in the extracellular matrix (Figure 6). Under these conditions, it was not possible to establish local gradients of QS signals or QQ enzymes, and there was little or no heterogeneity in the QS response of the system (Figures 4A and 5A). In fact, QQ had a limited effect on the QS behavior measured here with a 
low activation threshold of $1.5 \mathrm{nM}$. Nonetheless, a reduced, but global QQ effect, on QS signaling was predicted (Figures 4C vs 5C) and may therefore affect QS behaviors that rely on higher activation thresholds. These QS signaling and signalquenching interactions could be particularly relevant in environments where significant hydraulic retention time (HRT, i.e., water retention time) is expected, including water pockets in the rhizosphere, ${ }^{49}$ on wetted leaves, ${ }^{50}$ in sequencing batch bioreactors, ${ }^{19}$ in wastewater treatment plants, ${ }^{36}$ or even in the animal gastrointestinal tract. ${ }^{51}$ For example, a previous study comparing the quorum size of Pseudomonas syringae on dried and wetted leaves, indicated that the total population on the wetted leaves displayed at least $25 \%$ more QS activity compared to that of the dried condition after $48 \mathrm{~h}$ of incubation, ${ }^{50}$ suggesting that liquid/convection-mediated transport enables interaggregate communication. It is also likely that convection plays a critical role in mediating signal transduction in engineered ecosystems, such as in granular bioreactors where the suspended biofilm granules are constantly mixed by aeration, allowing signal exchange to occur between individual granules in the liquid medium. ${ }^{19}$ The observation that the EPS matrix absorbs signals from the environment (Figure 4E) provides a mechanistic insight into how biofilm aggregates effectively perform QS, even at extremely low signal concentrations (i.e., $\sim 500 \mathrm{pM}$ ) in a continuous suspended condition. ${ }^{19}$ In support of the role of the EPS in QS signaling, it has been shown that amyloid proteins (Fap), one of the key EPS matrices of Pseudomonas, bind reversibly AHLs (i.e., PQS, Pseudomonas quorum signals) in vitro. ${ }^{52}$ Further experiments using different EPS types, including EPS extracts from natural biofilm communities and QS signals are necessary to determine the role and specificity of the biofilm matrix in modulating $Q S$ activity in natural communities.

The experimental and modeling data show that QQ activity, in the hydrogel matrix, must be an extracellular/extra-aggregate process to have any significant impact on QS. This raises an important ecological question regarding the role of QQ in nature, as the majority of $\mathrm{QQ}$ isolates from different environments exhibit intracellular or cell-associated QQ activity, which is presumed to result from a lack of a signal peptide for secretion. ${ }^{53,54}$ Without secretion of the QQ enzyme, the cellular uptake and degradation of the environmental AHL by the QQ organism is probably too limited to compete with the signal production rates by other species. Therefore, our data suggest that the intracellular QQ enzymes may not have evolved primarily to degrade environmental AHL signals as a competition measure as previously proposed, ${ }^{55}$ at least not in habitats where the movement of QQ organisms is bound by the extracellular matrix. Instead, it is likely that the intracellular QQ enzymes have evolved to control other important cellular processes; for example, regulating the QS signal level in the AHL producers, ${ }^{56}$ acquiring QS signals as a nutrient source ${ }^{57}$ or for signal detoxification. $^{58}$ Alternatively, for $\mathrm{QQ}$ to evolve as a competitive advantage, high production rates of $Q Q$ enzymes are needed to counter the much higher population densities of co-occurring QS-producing organisms. We observed extracellular QQ which was also associated with the extracellular activity of alkaline phosphatase, an intracellular (periplasmic) enzyme. ${ }^{59}$ This suggests that cell lysis plays an important role in localized QQ activity and could be achieved by a subpopulation of cells undergoing lysis, as was observed for extracellular DNA (eDNA) release in Pseudomonas aerugino$s a .{ }^{60}$ While we did not detect QQ activity in the absence of cell lysis, it is also possible that the cytoplasmic AiiO may be released extracellularly via outer-membrane vesicles (OMVs). These OMVs are commonly used to excrete various cellular contents, including virulence factors and long-chain AHLs, in Gram-negative bacteria. ${ }^{61}$ However, future studies will be required to evaluate this hypothesis. The lack of a strong QQ effect for intracellular enzymes has significant implications for the development of QQ strains for biotechnological applications whereby QQ is used to control biofilm formation or other QS regulated behaviors. In contrast, stronger QQ effects would be expected if a secreted QQ enzyme was modeled. Indeed, the extent to which an extracellular QQ enzyme may impact on community $Q S$ could be readily simulated using our current model. While natural communities are more complex, we have explicitly designed this system to be as simple as possible to exclude erroneous factors such as differences in species growth rates, the production of competition molecules, differences in biofilm formation, maturation and dispersal, as well as differences in signal uptake and perception. In this way, the system described here was used to define the specific impacts of signal production, diffusion, recognition, and signal degradation on QS responses. The results presented here could thus help form the basis for studies of more complex, multispecies communities.

\section{ASSOCIATED CONTENT}

\section{sI Supporting Information}

The Supporting Information is available free of charge at https://pubs.acs.org/doi/10.1021/acs.est.0c00716.

Determination of QS activation thresholds in simulations and experiments; numerical model; encapsulation of bacteria in alginate hydrogel and characterizations of QS signal producer, quencher, and reporter strains; simulations of QS signaling in a hydrogel matrix without QQ leakage; extracellular QQ activity occurs through cell lysis; QS activation time mismatch between the experimental results and the simulations in a hydrogel matrix; parameter estimation for $Q S$ signaling in a hydrogel matrix in the presence of quorum quenchers; QS activation profiles of reporter aggregates; simulation of QS activation in a hydrogel matrix with 100-fold increased signal production rate; influence of air flow inside the enclosed chamber of the Gel-Doc XR+ system on the convective flow of an aqueous medium; influence of ambient air flow on convective flow of aqueous medium in Petri dish; relative fluorescence intensity of reporter aggregates exposed to different concentrations of AHL; scheme of the image quantification process; bacterial strains used in this study; and model parameter definitions (PDF)

\section{AUTHOR INFORMATION}

\section{Corresponding Authors}

Peter M. A. Sloot - Complexity Institute, Nanyang Technological University, 639798, Singapore; Institute for Advanced Study, University of Amsterdam, 1012 GC Amsterdam, The Netherlands; ITMO University, 197101 St. Petersburg, Russian Federation; Email: P.M.A.SLOOT@ UVA.NL 
Scott A. Rice - Singapore Centre for Environmental Life Sciences Engineering and The School of Biological Sciences, Nanyang Technological University, 637551, Singapore; The ithree Institute, University of Technology Sydney, 2007 Sydney, Australia; 이이.orid.org/0000-0002-9486-2343; Email: RSCOTT@NTU.EDU.SG

\section{Authors}

Chuan Hao Tan - Singapore Centre for Environmental Life Sciences Engineering and School of Materials Science and Engineering, Nanyang Technological University, 637551, Singapore

Hyun-Suk Oh - Singapore Centre for Environmental Life Sciences Engineering, Nanyang Technological University, 637551, Singapore; Department of Environmental Engineering, Seoul National University of Science and Technology, 01811 Seoul, South Korea; 이이이.org/0000-0002-9398-199X

Vivek M. Sheraton - Complexity Institute, Nanyang Technological University, 639798, Singapore

Emiliano Mancini - Institute for Advanced Study, University of Amsterdam, 1012 GC Amsterdam, The Netherlands

Say Chye Joachim Loo - Singapore Centre for Environmental Life Sciences Engineering and School of Materials Science and Engineering, Nanyang Technological University, 637551, Singapore; (1) orcid.org/0000-0001-5300-1275

Staffan Kjelleberg - Singapore Centre for Environmental Life Sciences Engineering and The School of Biological Sciences, Nanyang Technological University, 637551, Singapore; Centre for Marine Bio-Innovation, The Schools of Biotechnology and Biomolecular Sciences, and Biological, Earth and Environmental Sciences, University of New South Wales, 2031 Sydney, Australia

Complete contact information is available at: https://pubs.acs.org/10.1021/acs.est.0c00716

\section{Author Contributions \\ ${ }^{\dagger \dagger}$ C.H.T., H.-S.O., and V.M.S. contributed equally to this work. Notes}

The authors declare no competing financial interest.

\section{ACKNOWLEDGMENTS}

The authors would like to thank Dr Sharon Longford for helpful comments and discussion of this work. This research was supported by the Singapore Centre for Environmental Life Science Engineering (SCELSE), which is funded by the National Research Foundation Singapore, Ministry of Education, Nanyang Technological University and National University of Singapore, under its Research Centre of Excellence Programme.

\section{REFERENCES}

(1) Darch, S. E.; West, S. A.; Winzer, K.; Diggle, S. P. Densitydependent fitness benefits in quorum-sensing bacterial populations. Proc. Natl. Acad. Sci. U.S.A. 2012, 109, 8259-8263.

(2) Papenfort, K.; Bassler, B. L. Quorum sensing signal-response systems in Gram-negative bacteria. Nat. Rev. Microbiol. 2016, 14, 576-588.

(3) Jousset, A.; Eisenhauer, N.; Materne, E.; Scheu, S. Evolutionary history predicts the stability of cooperation in microbial communities. Nat. Commun. 2013, 4, 2573.

(4) Wang, M.; Schaefer, A. L.; Dandekar, A. A.; Greenberg, E. P. Quorum sensing and policing of Pseudomonas aeruginosa social cheaters. Proc. Natl. Acad. Sci. U.S.A. 2015, 112, 2187-2191.
(5) Dandekar, A. A.; Chugani, S.; Greenberg, E. P. Bacterial quorum sensing and metabolic incentives to cooperate. Science 2012, 338, 264-266.

(6) Whiteley, M.; Diggle, S. P.; Greenberg, E. P. Progress in and promise of bacterial quorum sensing research. Nature 2017, 551, $313-320$.

(7) Kirisits, M. J.; Margolis, J. J.; Purevdorj-Gage, B. L.; Vaughan, B.; Chopp, D. L.; Stoodley, P.; Parsek, M. R. Influence of the Hydrodynamic Environment on Quorum Sensing in Pseudomonas aeruginosa Biofilms. J. Bacteriol. 2007, 189, 8357-8360.

(8) Kim, M. K.; Ingremeau, F.; Zhao, A.; Bassler, B. L.; Stone, H. A. Local and global consequences of flow on bacterial quorum sensing. Nat. Microbiol. 2016, 1, 15005.

(9) Carnes, E. C.; Lopez, D. M.; Donegan, N. P.; Cheung, A.; Gresham, H.; Timmins, G. S.; Brinker, C. J. Confinement-induced quorum sensing of individual Staphylococcus aureus bacteria. Nat. Chem. Biol. 2010, 6, 41-45.

(10) Connell, J. L.; Wessel, A. K.; Parsek, M. R.; Ellington, A. D.; Whiteley, M.; Shear, J. B. Probing Prokaryotic Social Behaviors with Bacterial "Lobster Traps". mBio 2010, 1, No. e00202.

(11) Boedicker, J. Q.; Vincent, M. E.; Ismagilov, R. F. Microfluidic confinement of single cells of bacteria in small volumes initiates highdensity behavior of quorum sensing and growth and reveals its variability. Angew. Chem., Int. Ed. 2009, 48, 5908-5911.

(12) Tan, C. H.; Koh, K. S.; Xie, C.; Zhang, J.; Tan, X. H.; Lee, G. P.; Zhou, Y.; Ng, W. J.; Rice, S. A.; Kjelleberg, S. Community quorum sensing signalling and quenching: microbial granular biofilm assembly. npj Biofilms Microbiomes 2015, 1, 15006.

(13) Chopp, D. L.; Kirisits, M. J.; Moran, B.; Parsek, M. R. A mathematical model of quorum sensing in a growing bacterial biofilm. J. Ind. Microbiol. Biotechnol. 2002, 29, 339-346.

(14) Shompole, S.; Henon, K. T.; Liou, L. E.; Dziewanowska, K.; Bohach, G. A.; Bayles, K. W. Biphasic intracellular expression of Staphylococcus aureus virulence factors and evidence for Agrmediated diffusion sensing. Mol. Microbiol. 2003, 49, 919-927.

(15) Qazi, S. N. A.; Counil, E.; Morrissey, J.; Rees, C. E. D.; Cockayne, A.; Winzer, K.; Chan, W. C.; Williams, P.; Hill, P. J. agr Expression Precedes Escape of Internalized Staphylococcus aureus from the Host Endosome. Infect. Immun. 2001, 69, 7074-7082.

(16) Alberghini, S.; Polone, E.; Corich, V.; Carlot, M.; Seno, F.; Trovato, A.; Squartini, A. Consequences of relative cellular positioning on quorum sensing and bacterial cell-to-cell communication. FEMS Microbiol. Lett. 2009, 292, 149-161.

(17) Novick, R. P.; Geisinger, E. Quorum Sensing in Staphylococci. Annu. Rev. Genet. 2008, 42, 541-564.

(18) Oh, H.-S.; Lee, C.-H. Origin and evolution of quorum quenching technology for biofouling control in MBRs for wastewater treatment. J. Membr. Sci. 2018, 554, 331-345.

(19) Tan, C. H.; Koh, K. S.; Xie, C.; Tay, M.; Zhou, Y.; Williams, R.; $\mathrm{Ng}$, W. J.; Rice, S. A.; Kjelleberg, S. The role of quorum sensing signalling in EPS production and the assembly of a sludge community into aerobic granules. ISME J. 2014, 8, 1186-1197.

(20) Tan, C. H.; Koh, K. S.; Xie, C.; Zhang, J.; Tan, X. H.; Lee, G. P.; Zhou, Y.; Ng, W. J.; Rice, S. A.; Kjelleberg, S. Community quorum sensing signalling and quenching: microbial granular biofilm assembly. npj Biofilms Microbiomes 2015, 1, 15006.

(21) Dong, Y.-H.; Wang, L.-H.; Xu, J.-L.; Zhang, H.-B.; Zhang, X.-F.; Zhang, L.-H. Quenching quorum-sensing-dependent bacterial infection by an N-acyl homoserine lactonase. Nature 2001, 411, 813817.

(22) Molina, L. z.; Constantinescu, F.; Michel, L.; Reimmann, C.; Duffy, B.; DÃ@fago, G. v. Degradation of pathogen quorum-sensing molecules by soil bacteria: a preventive and curative biological control mechanism. FEMS Microbiol. Ecol. 2003, 45, 71-81.

(23) D’Angelo-Picard, C.; Faure, D.; Penot, I.; Dessaux, Y. Diversity of $\mathrm{N}$-acyl homoserine lactone-producing and-degrading bacteria in soil and tobacco rhizosphere. Environ. Microbiol. 2005, 7, 1796-1808.

(24) Muras, A.; López-Pérez, M.; Mayer, C.; Parga, A.; AmaroBlanco, J.; Otero, A. High prevalence of quorum-sensing and quorum- 
quenching activity among cultivable bacteria and metagenomic sequences in the Mediterranean sea. Genes 2018, 9, 100.

(25) Bjarnsholt, T.; Alhede, M.; Alhede, M.; Eickhardt-Sørensen, S. R.; Moser, C.; Kühl, M.; Jensen, P. Ø.; Høiby, N. The in vivo biofilm. Trends Microbiol. 2013, 21, 466-474.

(26) Stacy, A.; McNally, L.; Darch, S. E.; Brown, S. P.; Whiteley, M. The biogeography of polymicrobial infection. Nat. Rev. Microbiol. 2015, 14, 93-105.

(27) Decho, A. W.; Visscher, P. T.; Ferry, J.; Kawaguchi, T.; He, L.; Przekop, K. M.; Norman, R. S.; Reid, R. P. Autoinducers extracted from microbial mats reveal a surprising diversity of $\mathrm{N}$-acylhomoserine lactones (AHLs) and abundance changes that may relate to diel $\mathrm{pH}$. Environ. Microbiol. 2009, 11, 409-420.

(28) Flemming, H.-C.; Wingender, J.; Szewzyk, U.; Steinberg, P.; Rice, S. A.; Kjelleberg, S. Biofilms: an emergent form of bacterial life. Nat. Rev. Microbiol. 2016, 14, 563-575.

(29) Dilanji, G. E.; Langebrake, J. B.; De Leenheer, P.; Hagen, S. J. Quorum activation at a distance: spatiotemporal patterns of gene regulation from diffusion of an autoinducer signal. J. Am. Chem. Soc. 2012, 134, 5618-5626.

(30) Flickinger, S. T.; Copeland, M. F.; Downes, E. M.; Braasch, A. T.; Tuson, H. H.; Eun, Y.-J.; Weibel, D. B. Quorum sensing between Pseudomonas aeruginosa biofilms accelerates cell growth. J. Am. Chem. Soc. 2011, 133, 5966-5975.

(31) Connell, J. L.; Kim, J.; Shear, J. B.; Bard, A. J.; Whiteley, M. Real-time monitoring of quorum sensing in 3D-printed bacterial aggregates using scanning electrochemical microscopy. Proc. Natl. Acad. Sci. U.S.A. 2014, 111, 18255-18260.

(32) Darch, S. E.; Simoska, O.; Fitzpatrick, M.; Barraza, J. P.; Stevenson, K. J.; Bonnecaze, R. T.; Shear, J. B.; Whiteley, M. Spatial determinants of quorum signaling in a Pseudomonas aeruginosa infection model. Proc. Natl. Acad. Sci. U.S.A. 2018, 115, 4779-4784.

(33) Oh, H.-S.; Tan, C. H.; Low, J. H.; Rzechowicz, M.; Siddiqui, M. F.; Winters, H.; Kjelleberg, S.; Fane, A. G.; Rice, S. A. Quorum quenching bacteria can be used to inhibit the biofouling of reverse osmosis membranes. Water Res. 2017, 112, 29-37.

(34) Mohanty, A.; Tan, C. H.; Cao, B. Impacts of nanomaterials on bacterial quorum sensing: differential effects on different signals. Environ. Sci.: Nano 2016, 3, 351-356.

(35) Andersen, J. B.; Heydorn, A.; Hentzer, M.; Eberl, L.; Geisenberger, O.; Christensen, B. B.; Molin, S. r.; Givskov, M. gfpbased $\mathrm{N}$-acyl homoserine-lactone sensor systems for detection of bacterial communication. Appl. Environ. Microbiol. 2001, 67, 575585.

(36) Nielsen, P. H.; Mielczarek, A. T.; Kragelund, C.; Nielsen, J. L.; Saunders, A. M.; Kong, Y.; Hansen, A. A.; Vollertsen, J. A conceptual ecosystem model of microbial communities in enhanced biological phosphorus removal plants. Water Res. 2010, 44, 5070-5088.

(37) Almstrand, R.; Daims, H.; Persson, F.; Sörensson, F.; Hermansson, M. New methods for analysis of spatial distribution and coaggregation of microbial populations in complex biofilms. Appl. Environ. Microbiol. 2013, 79, 5978-5987.

(38) Stewart, P. S. Diffusion in biofilms. J. Bacteriol. 2003, 185, $1485-1491$.

(39) Czajkowski, R.; Krzyżanowska, D.; Karczewska, J.; Atkinson, S.; Przysowa, J.; Lojkowska, E.; Williams, P.; Jafra, S. Inactivation of AHLs by Ochrobactrum sp. A44 depends on the activity of a novel class of AHL acylase. Environ. Microbiol. Rep. 2011, 3, 59-68.

(40) Charlton, T. S.; De Nys, R.; Netting, A.; Kumar, N.; Hentzer, M.; Givskov, M.; Kjelleberg, S. A novel and sensitive method for the quantification of N-3-oxoacyl homoserine lactones using gas chromatography-mass spectrometry: application to a model bacterial biofilm. Environ. Microbiol. 2000, 2, 530-541.

(41) Hense, B. A.; Kuttler, C.; Müller, J.; Rothballer, M.; Hartmann, A.; Kreft, J.-U. Does efficiency sensing unify diffusion and quorum sensing? Nat. Rev. Microbiol. 2007, 5, 230-239.

(42) Redfield, R. Is quorum sensing a side effect of diffusion sensing? Trends Microbiol. 2002, 10, 365-370.
(43) Quiñones, B.; Dulla, G.; Lindow, S. E. Quorum sensing regulates exopolysaccharide production, motility, and virulence in Pseudomonas syringae. Mol. Plant-Microbe Interact. 2005, 18, 682693.

(44) Von Bodman, S. B.; Bauer, W. D.; Coplin, D. L. Quorum sensing in plant-pathogenic bacteria. Annu. Rev. Phytopathol. 2003, 41, $455-482$.

(45) Von Bodman, S. B.; Majerczak, D. R.; Coplin, D. L. A negative regulator mediates quorum-sensing control of exopolysaccharide production in Pantoea stewartii subsp. Proc. Natl. Acad. Sci. U.S.A. 1998, 95, 7687-7692.

(46) Diggle, S. P.; Griffin, A. S.; Campbell, G. S.; West, S. A. Cooperation and conflict in quorum-sensing bacterial populations. Nature 2007, 450, 411-414.

(47) Rumbaugh, K. P.; Trivedi, U.; Watters, C.; Burton-Chellew, M. N.; Diggle, S. P.; West, S. A. Kin selection, quorum sensing and virulence in pathogenic bacteria. Proc. R. Soc. B 2012, 279, 3584.

(48) Song, X.; Nobes, D. S. Experimental investigation of evaporation-induced convection in water using laser based measurement techniques. Exp. Therm. Fluid Sci. 2011, 35, 910-919.

(49) Carminati, A.; Moradi, A. B.; Vetterlein, D.; Vontobel, P.; Lehmann, E.; Weller, U.; Vogel, H.-J.; Oswald, S. E. Dynamics of soil water content in the rhizosphere. Plant Soil 2010, 332, 163-176.

(50) Dulla, G.; Lindow, S. E. Quorum size of Pseudomonas syringae is small and dictated by water availability on the leaf surface. Proc. Natl. Acad. Sci. U.S.A. 2008, 105, 3082-3087.

(51) Mudie, D. M.; Murray, K.; Hoad, C. L.; Pritchard, S. E.; Garnett, M. C.; Amidon, G. L.; Gowland, P. A.; Spiller, R. C.; Amidon, G. E.; Marciani, L. Quantification of gastrointestinal liquid volumes and distribution following a $240 \mathrm{~mL}$ dose of water in the fasted state. Mol. Pharm. 2014, 11, 3039-3047.

(52) Seviour, T.; Hansen, S. H.; Yang, L.; Yau, Y. H.; Wang, V. B.; Stenvang, M. R.; Christiansen, G.; Marsili, E.; Givskov, M.; Chen, Y.; Otzen, D. E.; Nielsen, P. H.; Geifman-Shochat, S.; Kjelleberg, S.; Dueholm, M. S. Functional amyloids keep quorum-sensing molecules in check. J. Biol. Chem. 2015, 290, 6457-6469.

(53) Fetzner, S. Quorum quenching enzymes. J. Biotechnol. 2015, 201, 2-14.

(54) Utari, P. D.; Vogel, J.; Quax, W. J. Deciphering physiological functions of AHL quorum quenching acylases. Front. Microbiol. 2017, $8,1123$.

(55) Dong, Y.-H.; Zhang, X.-F.; Xu, J.-L.; Zhang, L.-H. Insecticidal Bacillus thuringiensis silences Erwinia carotovora virulence by a new form of microbial antagonism, signal interference. Appl. Environ. Microbiol. 2004, 70, 954-960.

(56) Grandclément, C.; Tannières, M.; Moréra, S.; Dessaux, Y.; Faure, D. Quorum quenching: role in nature and applied developments. FEMS Microbiol. Rev. 2015, 40, 86-116.

(57) Flagan, S.; Ching, W.-K.; Leadbetter, J. R. Arthrobacter Strain VAI-A Utilizes Acyl-Homoserine Lactone Inactivation Products and Stimulates Quorum Signal Biodegradation by Variovorax paradoxus. Appl. Environ. Microbiol. 2003, 69, 909-916.

(58) Kaufmann, G. F.; Sartorio, R.; Lee, S.-H.; Rogers, C. J.; Meijler, M. M.; Moss, J. A.; Clapham, B.; Brogan, A. P.; Dickerson, T. J.; Janda, K. D. Revisiting quorum sensing: Discovery of additional chemical and biological functions for 3-oxo- $\mathrm{N}$-acylhomoserine lactones. Proc. Natl. Acad. Sci. U.S.A. 2005, 102, 309-314.

(59) Malamy, M. H.; Horecker, B. L. Release of alkaline phosphatase from cells of Escherichia coli upon lysozyme spheroplast formation. Biochemistry 1964, 3, 1889-1893.

(60) Whitchurch, C. B.; Tolker-Nielsen, T.; Ragas, P. C.; Mattick, J. $\mathrm{S}$. Extracellular DNA required for bacterial biofilm formation. Science 2002, 295, 1487.

(61) Schwechheimer, C.; Kuehn, M. J. Outer-membrane vesicles from Gram-negative bacteria: biogenesis and functions. Nat. Rev. Microbiol. 2015, 13, 605-619. 